Article

\title{
The Effect of the Addition of Fruit Powders on the Quality of Snacks with Jerusalem Artichoke during Storage
}

\author{
Agnieszka Kita ${ }^{1, *}$, Joanna Nowak ${ }^{1}$ and Anna Michalska-Ciechanowska ${ }^{2}$ (D) \\ 1 Department of Food Storage and Technology, Faculty of the Biotechnology and Food Science, \\ Wrocław University of Environmental and Life Sciences, Chełmońskiego 37, 51-630 Wrocław, Poland; \\ joanna.nowak@upwr.edu.pl \\ 2 Department of Fruit, Vegetable and Plant Nutraceutical Technology, Faculty of the Biotechnology and \\ Food Science, Wrocław University of Environmental and Life Sciences, Chełmońskiego 37, \\ 51-630 Wrocław, Poland; anna.michalska@upwr.edu.pl \\ * Correspondence: agnieszka.kita@upwr.edu.pl; Tel.: +48-71-3207-762
}

Received: 17 July 2020; Accepted: 11 August 2020; Published: 13 August 2020

\begin{abstract}
The aim of the study was to evaluate the physical, chemical and sensory properties of novel snacks gained from pellets composed of Jerusalem artichoke flour with the addition of cranberry, chokeberry and blackcurrant juice powders (after extrusion) fried or microwaved during 5-week storage. In ready-to-eat snacks stored in climatic chamber, the moisture content, texture, colour, total polyphenols content and antioxidant capacity, while in fat fraction of fried snacks-peroxide and acid values were examined. Overall sensory attributes were monitored as well. It was indicated that an addition of fruit powders increased the content of total polyphenols (on average by $40 \%$ ) and improved the antioxidant capacity of snacks obtained. The products with fruit powders exhibited more attractive colour, while their texture was harder (when cranberry and chokeberry powders were added) in comparison with the control sample. Snacks with fruit powders addition showed better storage stability, what was especially important in the case of fried snacks where oxidative deterioration is most significant. The best effect was observed when chokeberry or black currant powders were used as additives. Replacing frying by microwaving as expansion method allowed to obtain snacks with acceptable sensory attributes and good quality during storage.
\end{abstract}

Keywords: Helianthus tuberosus L.; frying; microwaves; snacks; fruit powders; storage; physicochemical properties

\section{Introduction}

Recently, consumers' interest in health-promoting snack products has significantly increased [1]. They are looking for new healthy products, which can be also obtained by modification of existed snacks by introducing changes in recipe as well as technology. One of the most popular group of snacks, mostly consumed by children and young people, are products obtained from extruded pellets later on expanded by frying. The pellets are gained from a variety of components in which starch-based substrates are dominant (including wheat, rice, corn or potato). In recent years, various ingredients, i.e., fruits, vegetables, whole grains and proteins have been incorporated into a formulation used for their preparation. The addition of these ingredients allows one to obtain extruded snacks with improved nutritional and antioxidant composition [2]. A new ingredient, rich in bioactive compounds, with high potential is Jerusalem artichoke tubers. It is a good source of carbohydrates, such as inulin and its derivatives, e.g., fructooligosaccharides [3]. Helianthus tuberosum L. is also a good source of numerous polyphenolic compounds [4]. Previously, more than 20 phenolic compounds have been 
identified in tubers among which chlorogenic acid was a dominant one [5]. Such composition makes the Jerusalem artichoke suitable for the obtainment of functional foods [6]. However, there were few attempts of using Jerusalem artichoke as an ingredient in snacks production. The artichoke flour was used as an additive in corn snacks (obtained by high temperature and short time of extrusion process) and the obtained products were highly ranged [7]. On the other hand, some undesired flavour changes of snacks were noticed. It was suggested that other ingredients may influence the sensory quality of these types of snacks.

From the technological point of view, snacks obtained from pellets are usually expanded by immersion in hot oil that significantly increases the fat content in ready-to-eat products as well as improve palatability. The fat content, which usually ranges between $20-40 \%$, depends on pellets composition, type of frying oil and frying conditions. Fat is not the only source of flavour and aroma, but it also makes a contribution to the texture and significantly increases the calorific value of the snacks. The quality of fat also influences the storage stability of fried products. Taking both aspects into consideration, some other additives with relatively strong antioxidative properties should be considered in design of modern snacks. Another option of novel snacks processing can be using of non-frying technologies that cause the expansion of products as an effect of application of high temperature without mass transfer. In such treatment, usually hot air or microwaves are used. The microwave heating is a technological process that has a great impact due to its efficiency in the expansion of snack foods [8]. However, the sensory properties of such snacks are modified to a different extent when compared to traditional technologies, thus, the comparison of these expansion methods on the quality of freshly prepared and stored snacks will provide a new insight in novel snacks preparation process. Previously, the concept of fruit powders addition was employed for the preparation of the extruded snacks [9], but only the basic chemical, physical properties and sensory attributes were considered. The products gained were characterised by lower fat and sugar contents and received good level of consumers acceptance which provide an inside into development of novel snacks with improved functional properties. Thus, the study aimed at the evaluation of physical and chemical properties in terms of polyphenolics and antioxidant capacity of novel snacks gained from pellets composed of Jerusalem artichoke flour with addition of fruit juice powders (after extrusion) fried or microwaved in order to compare their properties after 5-week storage.

\section{Materials and Methods}

\subsection{Materials}

Dried Jerusalem artichoke tuber in form of flour was obtained from a local producer (Poland). Dried potato products, i.e., starch was obtained from potato factory Pepees S.A. (Łomża, Poland) and trade grits were produced by a potato factory in Lublin (Poland). Corn semolina, salt and oil used as additives were purchased from the local market (Wrocław, Poland).

The blackcurrant $c v$. 'Ruben', cranberry $c v$. 'Pilgrim' and chokeberry fruits were gained from the experimental station Przybroda (Rokietnica near Poznań, Poland) grown according to the Integrated Fruit Production system (certificate of the Polish Main Inspectorate of Plant Health and Seed Inspection). Fruit juices were obtained using a hydraulic press (SRSE, Warsaw, Poland). Juices were mixed with $30 \%(w / w)$ of maltodextrin (Dextrose Equivalent (DE): 20-30; Hortimex, Poland) and spray dried using a B190 spray dryer (Buchi, Flawil, Switzerland) at an inlet temperature of $180^{\circ} \mathrm{C}$ and an outlet temperature of $70^{\circ} \mathrm{C}$ (feed flow rate: $400 \mathrm{~mL} / \mathrm{min}$ ). Powders were vacuum sealed and frozen at $-20^{\circ} \mathrm{C}$.

\subsection{Methods}

\subsubsection{Pellet Extrusion}

Material for pellets was extruded in a single-screw extruder (Brabender DN 20, Germany). The following extrusion parameters were kept constant: die size $(0.5 \mathrm{~mm} \times 80 \mathrm{~mm})$, speed of dough 
supplier (35 rpm), screw speed (120 rpm), screw loading (23 A) and barrel temperature distribution $\left(60^{\circ} \mathrm{C} / 70{ }^{\circ} \mathrm{C} / 80{ }^{\circ} \mathrm{C}\right)$. Strands that had previously been subjected to extrusion but were not expanded were cut into pellets of ca. $27 \times 27 \mathrm{~mm}$, dried at room temperature for $12 \mathrm{~h}$ to ca. $12-13 \%$ moisture content and sealed in polyethylene bags to equilibrate moisture until frying or expanding in microwave.

\subsubsection{Preparation of Snacks}

Snacks were obtained from pellets following $48 \mathrm{~h}$ of storage at room temperature. The samples were fried in hot $\left(180^{\circ} \mathrm{C}\right)$ rapeseed oil for $15-20 \mathrm{~s}$ ( $3 \mathrm{~s}$ after expanded product appears on the oil surface) (Beckers, Italy). The product to oil ration was kept at 1:20 w/v. The second method of obtaining snacks were microwaving at $700 \mathrm{~W}$ for $25 \mathrm{~s}$ (Amica, Poland). The snacks were packed into aluminium foil packets (100 g).

\subsubsection{Storage of Snacks}

The snacks (Figure 1) were stored under climatic chamber (Memmert, Germany) with conditions (temperature $40{ }^{\circ} \mathrm{C}$, relative air humidity (RH) $80 \%$, no access of light) for 5 weeks. It was assumed that one-week storage in climatic chamber would represent four weeks storage under standard conditions (temperature $20^{\circ} \mathrm{C}$, RH of $40-50 \%$ ). The temperature was constant during all storage time. Samples were collected for analyses immediately after manufacture and after every week of storage.
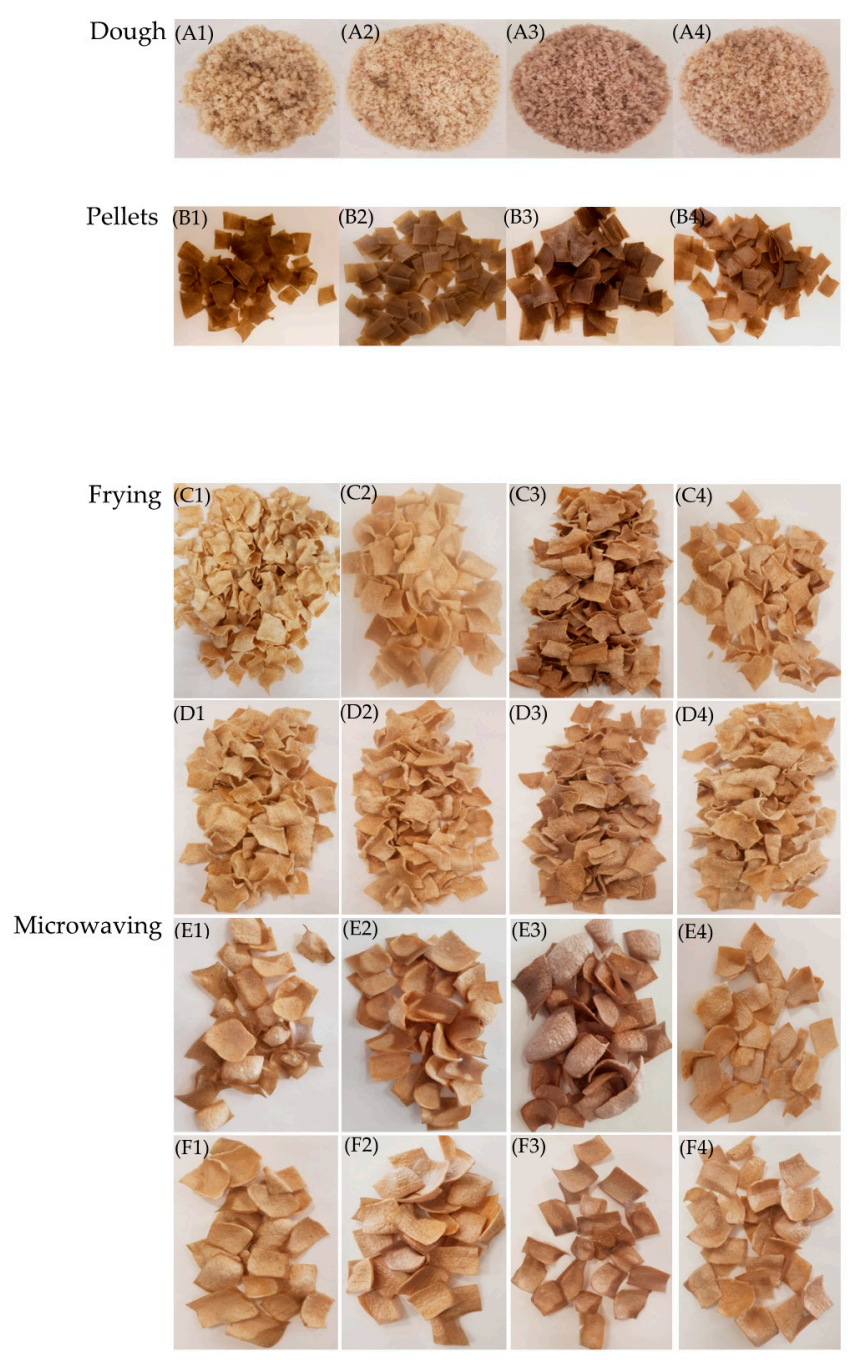
Figure 1. Processing of the obtainment of Jerusalem artichoke snacks. (A)—dough: (A1)—no fruit powder addition, (A2) — cranberry powder addition, (A3)—chokeberry powder addition, (A4)—blackcurrant powder addition; (B) — pellets: (B1)—no fruit powder addition, (B2)—cranberry powder addition, (B3) — chokeberry powder addition, (B4)—blackcurrant powder addition; Frying: (C) -0 days of storage and (D) -5 weeks of storage, (C1,D1) - no fruit powder addition, (C2,D2) — cranberry powder addition, (C3,D3) — chokeberry powder addition, (C4,D4)—blackcurrant powder addition; Microwaving: (E) -0 days of storage and $(\mathbf{F})-5$ weeks of storage, $(\mathbf{E} 1, \mathbf{F 1})-$ no fruit powder addition, (E2,F2) — cranberry powder addition, (E3,F3)—chokeberry powder addition, (E4,F4)—blackcurrant powder addition.

\subsubsection{Determination of Chemical Parameters}

Moisture content was determined by drying method [10]. Approximately $5 \mathrm{~g}$ of grounded samples were weighted and dried at $105{ }^{\circ} \mathrm{C}$ to a constant weight (for approximately $4 \mathrm{~h}$ ). Acid and peroxide values were determined in fat fraction of stored fried snacks after extraction by Folch method [11,12]. Results were reported as the arithmetic mean of three independent repetitions, taking into account the standard deviation.

\subsubsection{Texture}

The texture of obtained snacks was determined by using an Instron type 5544 texture-measuring device with Merlin software. The minimal force $(\mathrm{N})$ necessary to break up snacks was measured using a share blade at a displacement rate of $250 \mathrm{~mm} / \mathrm{min}$ [13].

\subsubsection{Colour}

The colour of snacks (Hunter lab scale) was measured using Minolta CM-5 spectrophotometer against a white reference standard. Hue angle and chroma colour space parameters were calculated from $a$ and $b$ values [14].

\subsubsection{Total Polyphenolics}

The total polyphenolics content was determined in $70 \%$ methanolic extracts $(w / w)$ using the Folin-Ciocalteu colorimetric method as described by Gao, Bjork, Trajkovski and Uggla [15]. The results $(n=2)$ were expressed as $\mu \mathrm{g}$ gallic acid equivalent (GAE) per $g$ of dry matter (DM).

\subsubsection{Antioxidant Capacity}

The antioxidant capacity was determined in $70 \%$ methanolic extracts $(w / w)$ by the Trolox equivalent antioxidant capacity (TEAC) by scavenging of ABTS ${ }^{\bullet+}$ radicals [16] and by FRAP (Ferric Reducing Antioxidant Potential) [17]. Results were expressed as $\mu \mathrm{mol}$ of Trolox equivalents per g DM. Data were expressed as an average of four measurements $(n=4)$.

\subsubsection{Sensory Attributes}

The sensory properties of obtained snacks were evaluated before and after 5-week storage in climatic chamber using a nine-degree hedonic scale with boundary indications: "I do not like it extremely" (1) to 'I like it extremely' (9). The assessment included the following quality attributes: texture, colour, taste, smell and overall acceptability. It was conducted by a group of 30 consumer panellists ( 15 men and 15 women at the age of 20-65). Coded samples were provided to the panellists for the evaluation at $20^{\circ} \mathrm{C}$ in uniform 50 -mL plastic containers [18].

\subsubsection{Statistical Analysis}

Statistical analyses of all data were performed using a double-way analysis of variance (ANOVA). Duncan's range test was used to determine the differences among samples with a probability level of 
$p \leq 0.05$. Statistical analyses and standard deviations were determined using Statistica v.13.3 software (StatSoft, Kraków, Poland).

\section{Results and Discussion}

\subsection{Physical Properties}

\subsubsection{Moisture Content}

Moisture content of snacks prepared with fruit powders differed due to the expansion method. Application of microwaves resulted in products with higher moisture content than that obtained after frying (Table 1). It can be explained by the fact that during frying the heat is transferred from the hot oil to the surface of the product by convection and by conduction from the surface of the snacks to their centre [19] within a relatively short period of time. The water present in fried product is moved from the centre to the surface and leave the products from this point as vapour. The moisture in the surface is trapped in the confined space and when it became superheated it altered the pore walls influencing the hardness of the samples [20]. On the other hand, during the microwave process the energy of microwaves is absorbed by water which creates a relatively high pressure in the centre of material subjected to drying leading to the rapid transfer of moisture [21], however, it was assumed that the temperature of the process was lower than frying. Previously, it was indicated that diffusion of water slows down at a lower temperatures, thus application of microwaves during snacks preparation may prevent from such intense moisture loss [22] leading to higher moisture content in the final products. Addition of fruit powders into the pellets' recipe also increased water content of obtained snacks. During storage moisture content increased in all analysed samples that was connected to water absorption. In general, snacks are very hygroscopic products and one of crucial element determining their storage stability is water content. According to different norms for fried snacks it should not be higher than $5 \%$ while for snacks with low fat content (as expanded by microwaves) - below $10 \%$ [23].

\subsubsection{Texture}

The texture of snacks was monitored by instrumental method when the hardness was measured by cutting of ready-to eat snacks. It was noted that the expansion method did not significantly influence this parameter (Table 1). In general, snacks should have a crunchy, delicate texture, without feeling hard $[23,24]$. Previously, it was noted that the introduction of fruit powders into pellets recipe increased the hardness of obtained snacks. Peksa et al. [25] noted that the addition of 5-30\% wheat bran increased the cutting force from $9.45 \mathrm{~N}$ to $22.77 \mathrm{~N}$. The influence of the additives on snacks hardness was also pointed by others [9]. In the current study, the hardness of snacks differed depending on the recipe. The control samples (no fruit powders addition) had the same hardness that snacks gained with the application of blackcurrant powder, while significantly harder texture had snacks with cranberry and chokeberry powders. Bhat et al. [26] indicated that the addition of tomato powder and raw lycopene to the recipe resulted in a significant increase in the hardness of the snacks compared to the control trial. The increase in the hardness of the snacks can be attributed to the increase in the fiber content of the snacks after the addition of tomato powder. On the other side, Peressini et al. [27] showed that the hardness of extruded snacks increased after the addition of soluble dietary fiber.

During storage, the hardness of all snacks analysed increased, however, values were not significantly different $(p \leq 0.05)$. It can be explained by the fact that such changes are usually connected with moisture changes of stored products. In described experiment snacks were protected by packaging material so both moisture as well as texture changes were minimalised. However, other studies suggested some changes in texture of stored products which were connected with some structural changes in stored material affected mostly by water content and interactions between different components. Raleng at al. [28] indicated a significant increase of hardness of deep-fried snacks with pineapple pomace powder addition after 2 months of storage performed at standard conditions. 
Table 1. Moisture content $(\%)$ and the texture $(\mathrm{N})$ of fried and microwaved snacks made from Jerusalem artichoke flour with addition of fruit powders during storage for 5 weeks.

\begin{tabular}{|c|c|c|c|c|c|c|c|c|}
\hline \multirow[t]{2}{*}{ Storage Time (Week) } & \multicolumn{2}{|c|}{ Control } & \multicolumn{2}{|c|}{ Cranberry } & \multicolumn{2}{|c|}{ Chokeberry } & \multicolumn{2}{|c|}{ Blackcurrant } \\
\hline & $\mathbf{F}$ & $\mathbf{M}$ & $\mathbf{F}$ & $\mathbf{M}$ & $\mathbf{F}$ & $\mathbf{M}$ & $\mathbf{F}$ & $\mathbf{M}$ \\
\hline \multicolumn{9}{|l|}{ Moisture (\%) } \\
\hline 0 & $3.42 \pm 0.03^{a, *}$ & $4.15 \pm 0.07^{\mathrm{b}, *}$ & $4.86 \pm 0.08^{\mathrm{d}, *}$ & $5.66 \pm 0.01^{\mathrm{f}, *}$ & $4.09 \pm 0.06^{\mathrm{b}, *}$ & $5.66 \pm 0.04^{\mathrm{f}, *}$ & $4.65 \pm 0.07^{\mathrm{c}, *}$ & $5.37 \pm 0.06^{\mathrm{e}, *}$ \\
\hline 1 & $3.55 \pm 0.07^{a, * *}$ & $4.19 \pm 0.04^{\mathrm{b}, * *}$ & $4.95 \pm 0.07^{\mathrm{d}, *}$ & $5.68 \pm 0.03^{f, *}$ & $4.48 \pm 0.06^{c, * *}$ & $5.69 \pm 0.01^{\mathrm{f}, *}$ & $4.76 \pm 0.01^{\mathrm{d}, *}$ & $5.39 \pm 0.01^{\mathrm{e}, *}$ \\
\hline 2 & $3.66 \pm 0.08^{a, * *}$ & $4.26 \pm 0.01 b^{b, * *}$ & $4.99 \pm 0.03^{\mathrm{d}, *}$ & $5.69 \pm 0.13^{f, *}$ & $4.52 \pm 0.03^{c, * *}$ & $5.70 \pm 0.14^{\mathrm{f}, *}$ & $4.97 \pm 0.03^{\mathrm{d}, * *}$ & $5.58 \pm 0.11^{\mathrm{e}, * *}$ \\
\hline 3 & $3.97 \pm 0.03^{a, * * *}$ & $4.61 \pm 0.01^{b, * * *}$ & $5.09 \pm 0.03^{\mathrm{d}, * *}$ & $5.86 \pm 0.01^{\mathrm{f}, * *}$ & $4.89 \pm 0.04^{c, * * *}$ & $5.78 \pm 0.05^{f, *}$ & $5.02 \pm 0.03^{\mathrm{d}, * *}$ & $5.62 \pm 0.03^{e, * *}$ \\
\hline 4 & $4.11 \pm 0.14^{\mathrm{a}, * * *}$ & $4.77 \pm 0.10^{\mathrm{b}, * * *}$ & $5.12 \pm 0.03^{d, * *}$ & $5.99 \pm 0.06^{\mathrm{f}, * * *}$ & $4.95 \pm 0.07^{c, * * *}$ & $6.02 \pm 0.03^{f, * *}$ & $5.08 \pm 0.06^{\mathrm{d}, * *}$ & $5.73 \pm 0.04^{\mathrm{e}, * *}$ \\
\hline 5 & $4.29 \pm 0.06^{a, * * * *}$ & $4.88 \pm 0.11^{b, * * * *}$ & $5.76 \pm 0.01^{\mathrm{d}, * * *}$ & $6.02 \pm 0.03^{e, * * *}$ & $5.00 \pm 0.12^{c, * * *}$ & $6.07 \pm 0.03^{\mathrm{e}, * *}$ & $5.24 \pm 0.03^{c, * * *}$ & $5.92 \pm 0.03^{e, * * *}$ \\
\hline \multicolumn{9}{|l|}{ Texture (N) } \\
\hline 0 & $17.00 \pm 9.17^{a, *}$ & $19.70 \pm 10.26^{\mathrm{a}, *}$ & $29.24 \pm 9.67^{c, *}$ & $28.11 \pm 17.46^{c, *}$ & $23.52 \pm 10.06^{b, *}$ & $24.61 \pm 5.50^{b, *}$ & $19.31 \pm 5.71^{\mathrm{a}, *}$ & $20.31 \pm 5.75^{\mathrm{a}, *}$ \\
\hline 1 & $17.36 \pm 7.25^{\mathrm{a}, *}$ & $20.51 \pm 5.46^{\mathrm{a}, *}$ & $29.51 \pm 9.68^{c, *}$ & $28.35 \pm 11.26^{c, *}$ & $23.73 \pm 10.14^{b, *}$ & $24.87 \pm 5.19^{b, *}$ & $19.71 \pm 5.44^{\mathrm{a}, *}$ & $21.11 \pm 7.25^{a, *}$ \\
\hline 2 & $17.87 \pm 10.25^{\mathrm{a}, *}$ & $21.55 \pm 20.52^{a, *}$ & $30.11 \pm 14.51^{c, *}$ & $29.64 \pm 20.52^{c, *}$ & $24.21 \pm 15.89^{b, *}$ & $25.37 \pm 12.24^{b, *}$ & $20.35 \pm 7.25^{a, *}$ & $22.03 \pm 16.22^{a, *}$ \\
\hline 3 & $18.20 \pm 7.95^{\mathrm{a}, *}$ & $21.93 \pm 12.98^{a, *}$ & $30.74 \pm 14.51^{\mathrm{c}, *}$ & $30.24 \pm 15.89^{c, *}$ & $24.87 \pm 19.47^{b, *}$ & $25.82 \pm 17.17^{b, *}$ & $20.75 \pm 9.18^{a, *}$ & $22.43 \pm 11.24^{\mathrm{a}, *}$ \\
\hline 4 & $18.61 \pm 8.58^{\mathrm{a}, *}$ & $22.09 \pm 7.65^{\mathrm{a}, *}$ & $31.34 \pm 30.79^{c, *}$ & $30.29 \pm 20.52^{c, *}$ & $25.35 \pm 14.51^{b, *}$ & $26.11 \pm 14.51^{b, *}$ & $21.20 \pm 12.98^{\mathrm{a}, *}$ & $22.99 \pm 8.83^{a, *}$ \\
\hline 5 & $19.05 \pm 10.48^{a, *}$ & $22.31 \pm 9.18^{a, *}$ & $31.70 \pm 13.04^{c, *}$ & $30.56 \pm 17.17^{c, *}$ & $25.73 \pm 12.98^{b, *}$ & $26.38 \pm 7.88^{b, *}$ & $21.64 \pm 11.24^{\mathrm{a}, *}$ & $23.34 \pm 10.26^{a, *}$ \\
\hline
\end{tabular}

F-fried, M-microwaved; values are means \pm standard deviation $(n=6)$; different letters $(\mathrm{a}, \mathrm{b}, \mathrm{c}, \mathrm{d}, \mathrm{e}, \mathrm{f})$ in rows and asterisk $\left(^{*}, * * * * * * * * *\right)$ in columns represent significant differences $(p \leq 0.05)$ 


\subsubsection{Colour}

The chromatic parameters $L^{*}, a^{*}, b^{*}, C$ and $h^{\circ}$ of snacks prepared without and with fruit powders addition are presented in Table 2 . In general, snacks gained after application of microwaves were lighter (average $L^{*}$ value of 75.6 ) in comparison to products obtained after frying (average $L^{*}$ value of 73.9), regardless the time of storage. Addition of chokeberry powder resulted in slight decrease in lightness, whereas addition of blackcurrant and cranberry juice powders caused an increase in $\mathrm{L}^{*}$ values of snacks gained after microwaving and frying. Storage did not cause a significant change in coordinate $L^{*}$ values in any type of snacks analysed. When the redness was concerned, snacks gained after frying (control and snacks with fruit powders addition) had almost 1.8-times higher values of coordinate $\mathrm{a}^{*}$ when compared to products obtained after application of microwaves. The most significant difference in redness between the type of processing applied for snacks obtainment was noted in case of control samples. It can be suggested that frying (stronger processing conditions) led at the same time to greater darkness $\left(\mathrm{L}^{*}\right)$ and to the generation of redness via Maillard reaction and caramelisation reactions that was previously described by Ngadi et al. [29] and Kerr [30]. Similar observation was made in case of frying and microwave cooking of Jerusalem Artichoke chips [31].

The addition of fruit powders resulted in an increase in $a^{*}$ values of, on average, $32 \%$ after application of microwaves. In the case of frying, the increase was only noticed when chokeberry and blackcurrant powders were used. No significant changes between the control sample and snacks gained with cranberry powder were observed which might suggest stronger degradation of the red components present in cranberry powders during frying.

The storage resulted in a slight increase in coordinate $\mathrm{a}^{*}$ values that was the strongest in case of control samples. This confirmed that progress of Maillard reaction and caramelisation reactions may occur also during the storage of snacks.

The increase in $b^{*}$ values was the strongest in case of control samples. In general, the yellowness of snacks was stronger after frying when compared to application of microwaves, irrespective to the addition of fruit powders into the pellets. This confirmed a strong impact of processing conditions on the probable occurrence of the newly formed compounds during snacks preparation [32]. In order to track further changes connected with influence of the processing in snacks, the examination of chroma $\left(C^{*}\right)$ indicating the colour intensity was done. In the case of the control samples, higher $C^{*}$ values were noticed for samples gained after frying. The addition of fruit powders resulted in an increase in chroma values, except chokeberry. Only in this case, the colour density was the lowest. Similar observation was done for snacks gained after application of microwaves. The hue angle $\left(\mathrm{h}^{\circ}\right)$ is defined as a quality attribute of colour [32]. For example, an angel of $0^{\circ}$ represents red hue, whereas angle of $90^{\circ}$ indicate yellow. When the non-stored snacks were concerned, slightly more reddish products were gained after addition of chokeberry powders, whereas the most yellowish were the control samples. No significant changes were noted in $\mathrm{h}^{\mathrm{o}}$ values between snacks prepared after application of microwaves and frying, except samples with chokeberry powder. In this case, the frying led to the decrease in $h^{o}$ values, thus the samples were more reddish than that gained after application of microwaves. Storage did not cause significant changes in $\mathrm{h}^{\mathrm{o}}$ values, irrespective to the type of powder used. 
Table 2. Colour $\left(L, a^{*}, b^{*}, C, h^{\circ}\right)$ and browning index of fried and microwaved snacks made from Jerusalem artichoke flour with addition of fruit powders during storage for 5 weeks.

\begin{tabular}{|c|c|c|c|c|c|c|c|c|}
\hline \multirow[t]{2}{*}{ Storage Time (Week) } & \multicolumn{2}{|c|}{ Control } & \multicolumn{2}{|c|}{ Cranberry } & \multicolumn{2}{|c|}{ Chokeberry } & \multicolumn{2}{|c|}{ Blackcurrant } \\
\hline & $\mathbf{F}$ & $\mathbf{M}$ & $\mathbf{F}$ & $\mathbf{M}$ & F & M & F & $\mathbf{M}$ \\
\hline \multicolumn{9}{|l|}{$L^{*}$} \\
\hline 0 & $67.44 \pm 0.03^{c, *}$ & $76.09 \pm 0.07^{\mathrm{g}, *}$ & $68.12 \pm 0.08^{\mathrm{d}, *}$ & $77.59 \pm 0.07^{\mathrm{h}, *}$ & $56.03 \pm 0.05^{\mathrm{a}, *}$ & $71.35 \pm 0.13^{\mathrm{e}, *}$ & $61.64 \pm 0.03^{b, *}$ & $75.31 \pm 0.05^{\mathrm{f}, *}$ \\
\hline 1 & $67.58 \pm 0.01^{c, *}$ & $76.54 \pm 0.03^{g, *}$ & $68.52 \pm 0.01^{\mathrm{d}, *}$ & $77.62 \pm 0.01^{d_{,} *}$ & $56.66 \pm 0.04^{\mathrm{a}, * * *}$ & $72.43 \pm 0.02^{\mathrm{e}, * *}$ & $61.87 \pm 0.01^{b, *}$ & $75.38 \pm 0.00^{\mathrm{f}, *}$ \\
\hline 2 & $67.76 \pm 0.09^{c, *}$ & $76.62 \pm 0.01^{g}, * ; * *$ & $68.90 \pm 0.08 \mathrm{~A}^{\mathrm{d}, *, * *}$ & $77.69 \pm 0.01 \mathrm{~d}, *$ & $56.73 \pm 0.07^{\mathrm{a}, * *}$ & $72.45 \pm 0.04 \mathrm{e}^{\mathrm{e}, * *}$ & $61.93 \pm 0.03^{b, *}$ & $75.41 \pm 0.07^{\mathrm{f}, *}$ \\
\hline 3 & $68.03 \pm 0.02^{c, * *}$ & $76.83 \pm 0.02^{g, * *}$ & $69.16 \pm 0.04 \mathrm{~d}, * *$ & $77.74 \pm 0.03^{\mathrm{d}, *}$ & $56.81 \pm 0.01^{\mathrm{a}, * *}$ & $72.52 \pm 0.01 \mathrm{e}^{\mathrm{e}, * *}$ & $61.99 \pm 0.01^{b, *}$ & $75.52 \pm 0.01^{\mathrm{f}, *}$ \\
\hline 4 & $68.33 \pm 0.02^{c, * *}$ & $76.88 \pm 0.03 \mathrm{~g}, * * *$ & $69.85 \pm 0.08^{d, * * *}$ & $77.81 \pm 0.01 \mathrm{~d}, *$ & $56.97 \pm 0.01^{\mathrm{a}, * *}$ & $72.59 \pm 0.01^{\mathrm{e}, * *}$ & $62.06 \pm 0.04^{b, *}$ & $75.73 \pm 0.02^{\mathrm{f}, * * * *}$ \\
\hline 5 & $68.67 \pm 0.05^{c, * *}$ & $77.00 \pm 0.07 \mathrm{~g}, * * *$ & $70.17 \pm 0.07^{\mathrm{d}, * * * *}$ & $77.88 \pm 0.06^{\mathrm{d}, *}$ & $57.31 \pm 0.01^{a_{1, * * *}}$ & $72.63 \pm 0.02^{e, * *}$ & $62.38 \pm 0.06^{b, * *}$ & $75.97 \pm 0.05^{\mathrm{f}, * *}$ \\
\hline \multicolumn{9}{|c|}{ (1) } \\
\hline 0 & $4.01 \pm 0.01^{\mathrm{e}, *}$ & $1.76 \pm 0.04^{\mathrm{a}, *}$ & $3.91 \pm 0.01 \mathrm{~d}, *$ & $2.62 \pm 0.01^{c, *}$ & $5.43 \pm 0.02 \mathrm{~g}, *$ & $2.48 \pm 0.06^{\mathrm{b}, *}$ & $5.06 \pm 0.04^{\mathrm{f}, *}$ & $2.66 \pm 0.04^{c, *}$ \\
\hline 1 & $4.10 \pm 0.07^{\mathrm{e}, * *}$ & $1.77 \pm 0.02^{a, *}$ & $3.99 \pm 0.01 \mathrm{~d}, * * *$ & $2.65 \pm 0.05^{c, *}$ & $5.45 \pm 0.04 \mathrm{~g}, *$ & $2.52 \pm 0.01^{b, *}$ & $5.15 \pm 0.01^{\mathrm{f}, * *}$ & $2.67 \pm 0.02^{c, *}$ \\
\hline 2 & $4.17 \pm 0.07^{\mathrm{e}, * * *}$ & $1.80 \pm 0.06^{a, * *}$ & $4.15 \pm 0.03 \mathrm{~d}, * * * *$ & $2.68 \pm 0.06^{b, c, *}$ & $5.66 \pm 0.03 \mathrm{~g}, * *$ & $2.55 \pm 0.02^{b, *, * *}$ & $5.27 \pm 0.02^{\mathrm{f}, * * *}$ & $2.70 \pm 0.07^{c, *}$ \\
\hline 3 & $4.31 \pm 0.01 \mathrm{e}, * * * * *$ & $1.86 \pm 0.06^{a, * *}$ & $4.29 \pm 0.02^{\mathrm{d}, * * * *}$ & $2.69 \pm 0.07 b, c, *$ & $5.87 \pm 0.02 \mathrm{~g}, * * * *$ & $2.65 \pm 0.05^{b, * * *}$ & $5.31 \pm 0.02^{\mathrm{f}, * * *}$ & $3.18 \pm 0.06^{c, * *}$ \\
\hline 4 & $4.41 \pm 0.01^{\mathrm{e}, * * * * *}$ & $1.94 \pm 0.04^{\mathrm{a}, * * *}$ & $4.51 \pm 0.01^{\mathrm{d}, * * * * *}$ & $2.70 \pm 0.10^{c, * *}$ & $6.09 \pm 0.06^{g, * * * *}$ & $2.97 \pm 0.02^{b, * * * *}$ & $5.35 \pm 0.01^{\mathrm{f}, * * * * * * * *}$ & $3.20 \pm 0.07^{c, * *}$ \\
\hline 5 & $4.55 \pm 0.05^{e^{* * * * * * *}}$ & $1.98 \pm 0.01^{\mathrm{a}, * * *}$ & $4.63 \pm 0.02^{\mathrm{d}, * * * * * *}$ & $2.82 \pm 0.02^{c, * *}$ & $6.17 \pm 0.01 \mathrm{~g}, * * * * * *$ & $3.00 \pm 0.07^{b, * * * *}$ & $5.41 \pm 0.01^{\mathrm{f}, * * * *}$ & $3.37 \pm 0.01^{c, * * *}$ \\
\hline \multicolumn{9}{|c|}{ - } \\
\hline 0 & $22.20 \pm 0.04 \mathrm{~g}, *$ & $15.11 \pm 0.12^{b, *}$ & $21.35 \pm 0.03^{\mathrm{f}, *}$ & $15.81 \pm 0.07^{\mathrm{d}, *}$ & $15.24 \pm 0.03^{c, *}$ & $11.16 \pm 0.06^{\mathrm{a}, *}$ & $18.72 \pm 0.01 \mathrm{e}, *$ & $14.30 \pm 0.07^{b, *}$ \\
\hline 1 & $22.48 \pm 0.06^{g, * *}$ & $15.58 \pm 0.06^{\mathrm{b}, * *}$ & $21.42 \pm 0.02^{\mathrm{f}, * *}$ & $15.82 \pm 0.06^{d_{, *}}$ & $16.23 \pm 0.03^{c, * *}$ & $11.90 \pm 0.07^{\mathrm{a}, * *}$ & $18.86 \pm 0.01^{\mathrm{e}, * *}$ & $14.39 \pm 0.06^{\mathrm{b}, * *}$ \\
\hline 2 & $22.56 \pm 0.03 \mathrm{~g}, * * *$ & $15.78 \pm 0.01^{b, * * *}$ & $21.79 \pm 0.05^{\mathrm{f}, * * *}$ & $15.92 \pm 0.01 \mathrm{~d}, * *$ & $16.76 \pm 0.03^{c, * * *}$ & $12.13 \pm 0.02^{a, * * *}$ & $19.40 \pm 0.07 \mathrm{e}, * * *$ & $14.47 \pm 0.05^{b, * * *}$ \\
\hline 3 & $22.72 \pm 0.01 \mathrm{~g}, * * * *$ & $16.20 \pm 0.07^{b, * * * *}$ & $21.89 \pm 0.07^{\mathrm{f}, * * * *}$ & $16.43 \pm 0.07^{\mathrm{d}, * * *}$ & $16.78 \pm 0.06^{c, * * *}$ & $12.25 \pm 0.04^{\mathrm{a}, * * * *}$ & $19.63 \pm 0.02^{\mathrm{e}, * * * *}$ & $14.53 \pm 0.02^{b, * * *}$ \\
\hline 4 & $22.83 \pm 0.08 \mathrm{~g}, * * * * * *$ & $16.75 \pm 0.04^{\mathrm{b}, * * * * *}$ & $21.91 \pm 0.01^{\mathrm{f}, * * * *}$ & $16.87 \pm 0.05^{\mathrm{d}, * * * *}$ & $16.85 \pm 0.04^{c, * * * *}$ & $12.91 \pm 0.01^{\mathrm{a}, * * * * *}$ & $19.74 \pm 0.03^{\mathrm{e}, * * * * *}$ & $14.83 \pm 0.02^{b, * * * *}$ \\
\hline 5 & $23.12 \pm 0.01 \mathrm{~g}, * * * * * * *$ & $16.86 \pm 0.04^{b, * * * * * *}$ & $22.34 \pm 0.03^{\mathrm{f}, * * * * *}$ & $16.90 \pm 0.07^{\mathrm{d}, * * * *}$ & $16.97 \pm 0.02^{c, * * * * *}$ & $12.96 \pm 0.01^{\mathrm{a}, * * * * *}$ & $19.88 \pm 0.07^{\mathrm{e}^{* * * * * * *}}$ & $14.98 \pm 0.02^{b, * * * * * *}$ \\
\hline \multicolumn{9}{|l|}{ Chroma } \\
\hline 0 & $22.61 \pm 0.04^{\mathrm{h}, *}$ & $15.20 \pm 0.07^{c, *}$ & $21.57 \pm 0.05^{g}, *$ & $16.03 \pm 0.07^{\mathrm{d}_{*} *}$ & $16.41 \pm 0.03^{\mathrm{e}, *}$ & $11.25 \pm 0.04^{\mathrm{a}, *}$ & $19.03 \pm 0.52^{\mathrm{f}, *}$ & $14.74 \pm 0.06^{\mathrm{b}, *}$ \\
\hline 1 & $23.19 \pm 0.04^{\mathrm{h}, * *}$ & $15.54 \pm 0.03^{c, * *}$ & $21.88 \pm 0.06^{\mathrm{g}, * *}$ & $16.53 \pm 0.03^{\mathrm{d}, * *}$ & $17.00 \pm 0.07^{\mathrm{e}, * *}$ & $11.52 \pm 0.02^{\mathrm{a}, * *}$ & $19.48 \pm 0.01^{\mathrm{f}, * *}$ & $14.74 \pm 0.06^{\mathrm{b}, *}$ \\
\hline 2 & $23.21 \pm 0.07^{\mathrm{h}, * *}$ & $15.69 \pm 0.06^{c, * *}$ & $22.18 \pm 0.05^{g, * * *}$ & $16.98 \pm 0.01^{\mathrm{d}, * * *}$ & $17.16 \pm 0.04^{\mathrm{e}, * * *}$ & $11.90 \pm 0.35^{a, * * *}$ & $20.14 \pm 0.03^{f, * * *}$ & $14.79 \pm 0.06^{\mathrm{b}, *}$ \\
\hline 3 & $23.32 \pm 0.02 \mathrm{~h}, * *$ & $16.13 \pm 0.02^{c, * * *}$ & $22.47 \pm 0.05^{g, * * * *}$ & $17.21 \pm 0.07^{d, * * * * *}$ & $17.27 \pm 0.08^{\mathrm{e}, * * *}$ & $11.98 \pm 0.01^{\mathrm{a}, * * *}$ & $20.33 \pm 0.03^{\mathrm{f}, * * * *}$ & $14.86 \pm 0.04^{\mathrm{b}, *}$ \\
\hline 4 & $23.35 \pm 0.08^{\mathrm{h}, * *, * * *}$ & $16.87 \pm 0.07^{c, * * * *}$ & $22.63 \pm 0.08 \mathrm{~g}, * * * * * *$ & $17.06 \pm 0.04 \mathrm{~d}, * * * *$ & $17.39 \pm 0.04^{\mathrm{e}, * * * *}$ & $12.29 \pm 0.10^{\mathrm{a}, * * * *}$ & $20.45 \pm 0.03^{\mathrm{f}, * * * *}$ & $15.87 \pm 0.10^{\mathrm{b}, * *}$ \\
\hline 5 & $23.46 \pm 0.04^{\mathrm{h}, * * *}$ & $16.90 \pm 0.07^{c, * * * *}$ & $22.78 \pm 0.05 \mathrm{~g}, * * * * * *$ & $17.25 \pm 0.04^{\mathrm{d}, * * * * * *}$ & $17.46 \pm 0.06^{\mathrm{e}, * * * *}$ & $12.43 \pm 0.02^{\mathrm{a}, * * * * *}$ & $20.57 \pm 0.05^{\mathrm{f}, * * * *, * * * * *}$ & $15.92 \pm 0.02^{b, * *}$ \\
\hline \multicolumn{9}{|c|}{ - } \\
\hline 0 & $79.02 \pm 0.05^{\mathrm{f}, *}$ & $82.02 \pm 0.02^{\mathrm{h}, *}$ & $78.26 \pm 0.04^{\mathrm{e}, *}$ & $80.34 \pm 0.03 \mathrm{~g}, *$ & $68.24 \pm 0.05^{\mathrm{a}, *}$ & $75.63 \pm 0.02^{c, *}$ & $74.25 \pm 0.15^{b, *}$ & $77.26 \pm 0.04^{\mathrm{d}, *}$ \\
\hline 1 & $79.29 \pm 0.08^{\mathrm{f}, * *}$ & $82.71 \pm 0.07 \mathrm{~h}, * *$ & $78.25 \pm 0.17^{\mathrm{e}, *}$ & $80.59 \pm 0.11 \mathrm{~g}, * *$ & $68.65 \pm 0.04^{a, * *}$ & $75.85 \pm 0.04^{c, * *}$ & $75.45 \pm 0.06^{\mathrm{b}, * *}$ & $77.38 \pm 0.06^{\mathrm{d}, * *}$ \\
\hline 2 & $79.94 \pm 0.01^{\mathrm{f}, * * *}$ & $82.85 \pm 0.04^{\mathrm{h}, * *}$ & $78.61 \pm 0.07 \mathrm{e}, * *$ & $80.61 \pm 0.07 \mathrm{~g}, * *$ & $68.95 \pm 0.06^{\mathrm{a}, * * *}$ & $76.16 \pm 0.1^{c, * * *}$ & $75.56 \pm 0.02^{b, * * *}$ & $77.49 \pm 0.07^{\mathrm{d}, * * *}$ \\
\hline 3 & $79.98 \pm 0.06^{\mathrm{f}, * * *}$ & $82.93 \pm 0.19^{\mathrm{h}, * *, * * * *}$ & $79.21 \pm 0.10^{\mathrm{e}, * * *}$ & $80.68 \pm 0.07 \mathrm{~g}, * *$ & $68.96 \pm 0.02^{a}, * * * *$ & $76.20 \pm 0 .{ }^{c, * * *}$ & $75.68 \pm 0.01^{b, * * *}$ & $77.50 \pm 0.07^{d, * * *}$ \\
\hline 4 & $80.19 \pm 0.06^{\mathrm{f}, * * * *}$ & $83.47 \pm 0.05^{\mathrm{h}, * * * *}$ & $79.55 \pm 0.04^{\mathrm{e}, * * * *}$ & $81.27 \pm 0.05^{g, * * *}$ & $68.98 \pm 0.01^{a, * * *}$ & $76.21 \pm 0.01^{\mathrm{c}, * * * * * * * *}$ & $75.82 \pm 0.02^{b, * * * *}$ & $77.58 \pm 0.06^{\mathrm{d}, * * * *}$ \\
\hline 5 & $80.29 \pm 0.07^{\mathrm{f}, * * * * *}$ & $83.57 \pm 0.05^{\mathrm{h}, * * * * *}$ & $79.78 \pm 0.06^{\mathrm{e}, * * * * *}$ & $81.35 \pm 0.04 \mathrm{~g}, * * *$ & $69.04 \pm 0.03^{a, * * * *}$ & $76.23 \pm 0.03^{c, * * *}$ & $75.97 \pm 0.01^{b, * * * * *}$ & $77.76 \pm 0.04^{d_{, * * * *}}$ \\
\hline
\end{tabular}


Table 2. Cont.

\begin{tabular}{|c|c|c|c|c|c|c|c|c|}
\hline \multirow[t]{2}{*}{ Storage Time (Week) } & \multicolumn{2}{|c|}{ Control } & \multicolumn{2}{|c|}{ Cranberry } & \multicolumn{2}{|c|}{ Chokeberry } & \multicolumn{2}{|c|}{ Blackcurrant } \\
\hline & F & $\mathbf{M}$ & $\mathbf{F}$ & $\mathbf{M}$ & $\mathbf{F}$ & $\mathbf{M}$ & $\mathbf{F}$ & $\mathbf{M}$ \\
\hline \multicolumn{9}{|l|}{ Browning index } \\
\hline 0 & $43.5 \pm 0.1^{\mathrm{f}, *}$ & $23.4 \pm 0.2^{b, *}$ & $41.1 \pm 0.2^{\mathrm{e}, *} \mathrm{AA}$ & $24.8 \pm 0.1 \mathrm{Ac}^{\mathrm{c}, *}$ & $38.3 \pm 0.1^{\mathrm{d}, *}$ & $19.2 \pm 0.1^{\mathrm{a}, *}$ & $41.6 \pm 0.0 \mathrm{e}$ & $23.2 \pm 0.1^{b, *}$ \\
\hline 1 & $44.1 \pm 0.2^{\mathrm{e}, * *}$ & $24.0 \pm 0.1^{c, * *}$ & $41.0 \pm 0.1 \mathrm{~d}, *$ & $24.8 \pm 0.2^{\mathrm{c}, *}$ & $40.3 \pm 0.0^{\mathrm{d}, * *}$ & $20.1 \pm 0.3^{a, * *}$ & $41.8 \pm 0.2 \mathrm{~d}, *$ & $23.4 \pm 0.2^{b, *}$ \\
\hline 2 & $44.2 \pm 0.1^{g, * *}$ & $24.3 \pm 0.1^{c, * *}$ & $41.7 \pm 0.3^{\mathrm{e}, * *}$ & $25.1 \pm 0.1^{\mathrm{d}, *, * *}$ & $41.8 \pm 0.1 \mathrm{e}, * * * *$ & $20.5 \pm 0.2^{\mathrm{a}, * *}$ & $43.2 \pm 0.0^{\mathrm{f}, * * *}$ & $23.5 \pm 0.2^{b, *}$ \\
\hline 3 & $44.5 \pm 0.1 \mathrm{~g}, * *$ & $25.0 \pm 0.1^{c, * * *}$ & $41.9 \pm 0.1^{\mathrm{e}, * *}$ & $25.8 \pm 0.0^{\mathrm{d}, * * *}$ & $42.0 \pm 0.2^{\mathrm{e}, * * *}$ & $20.8 \pm 0.1^{a, * *,, * * *}$ & $43.7 \pm 0.1^{\mathrm{f}, * *}$ & $24.0 \pm 0.1^{b, * *}$ \\
\hline 4 & $44.6 \pm 0.1^{g, * *}$ & $25.9 \pm 0.1^{c, * * * *}$ & $41.7 \pm 0.1^{\mathrm{e}, * *}$ & $26.5 \pm 0.1^{\mathrm{d}, * * * *}$ & $42.4 \pm 0.03^{\mathrm{f}, * * * *}$ & $22.2 \pm 0.1^{\mathrm{a}, * * * *}$ & $43.9 \pm 0.1^{\mathrm{f}, * * *}$ & $24.5 \pm 0.1^{b, * * *}$ \\
\hline 5 & $45.1 \pm 0.2^{f, * * * *}$ & $26.1 \pm 0.1^{b, * * * *}$ & $42.4 \pm 0.1^{\mathrm{e}, * * *}$ & $26.6 \pm 0.1^{c, * * * *}$ & $42.5 \pm 0.01^{\mathrm{e}, * * * *}$ & $22.3 \pm 0.2^{a, * * * * *}$ & $41.6 \pm 0.1^{\mathrm{d}, *}$ & $26.8 \pm 0.0^{c, * * * *}$ \\
\hline
\end{tabular}

$\mathrm{F}-$ fried, $\mathrm{M}-$ microwaved; values are means \pm standard deviation $(n=6)$; different letters $(\mathrm{a}, \mathrm{b}, \mathrm{c}, \mathrm{d}, \mathrm{e}, \mathrm{f}, \mathrm{g}, \mathrm{h})$ in rows and asterisk $\left(^{*}, * * * * *, * * * *\right)$ in columns represent significant differences $(p \leq 0.05)$. 


\subsubsection{Browning Index (BI)}

The browning index was performed in order to indicate the non-enzymatic alterations in browning colour of the samples [32]. In the case of analysed snacks, the lowest values were noted for control samples, regardless of the way if their obtainment (frying, microwaves). In general, snacks gained after frying had almost 1.8-fold higher browning index than products obtained after application of microwaves, regardless of the storage time (Table 2). This points out a strong influence of the processing on non-enzymatic changes in snacks. When the type of fruit powders was considered, the value of browning index was lower when the chokeberry and blackcurrant powders was added to fried and microwaved snacks when compared to control sample (no fruit powder addition). Taking the above into consideration, the application of natural fruit-based powders might prevent the formation of browning compounds. Storage also had an impact on browning index values. In general, browning index values increased, on average, about $10 \%$ after 5 weeks of storage and the increase was linear within that time.

A strong positive correlation between browning index (BI) and $a^{*}$ and $b^{*}$ values $(\mathrm{r}=0.85$ and $r=0.85$, respectively) was noted that pointed a possible formation of Maillard reaction and caramelisation products in microwave irradiated and fried samples. This was confirmed by positive correlation between BI and $C^{*}$ indicating the strength of processing $(r=0.87)$. No correlation was indicated between $\mathrm{BI}$ and $L^{*}$.

\subsection{Chemical Properties}

\subsubsection{Total Polyphenols}

For the non-stored samples, the content of total polyphenols in control samples (without fruit powders addition) was approximately $7 \%$ higher in products gained after usage of microwaves than in fried ones (Table 3). This pointed out an influence of the processing type on the thermolability of native polyphenols present in snacks.

Fruit powders added to pellets $(3 \% ; w / w)$ were obtained from blackcurrant, chokeberry and cranberry spray-dried juice gained with addition of maltodextrin. The dominant groups of polyphenols in blackcurrant powders were: anthocyanins (cyanidin- and delphinidin-3-O-rutinoside being the most abundant), flavan-3-ols, flavonols and phenolic acids [33], whereas in chokeberry five major groups of polyphenolics were identified: phenolic acids, anthocyanins, flavonols, flavan-3-ols and procyanidins (UPLC analyses; data not shown). On the other hand, in cranberry juice powders the dominant groups were: flavonols, followed by anthocyanins, phenolic acids and flavan-3-ols (UPLC analyses; data not shown). The addition of fruit juice powders into pellets resulted in an increase in total polyphenols content in following order: chokeberry (approximately increase of $29.8 \%$ ), cranberry (approximately 26.4\%) and blackcurrant (approximately 23\%) for products gained after application of microwaves and in the same order but to a slightly lower extent $(26.8 ; 26.7$ and $15.9 \%$, respectively) in fried products when compare to control samples (Table 3). This showed an influence of the processing type on the thermolability of polyphenols originated from fruit juice powders in snacks and proved their better retention after application of microwaves used for snacks preparation. It was indicated that the microwave vacuum drying process resulted in better retention of selected polyphenolic compounds during dehydration of plum products [33,34], thus, this method can be recommended for the preparation of products with improved functional properties. Previously, Camire et al. [35] tested addition of commercial spray-dried powders into extruded corn breakfast cereals and it was observed that usage of selected types of fruit powders improved the total polyphenolics contents in products analysed and differed in terms of the chemical composition of fruits. Storage caused a linear decrease in total polyphenols contents that after 5 weeks was $27.5 \%$ lower in case of microwaved and $40.4 \%$ for fried products when compared to control samples. This indicated that application of microwaves for snacks preparation seems to be more suitable for preservation of bioactive components in such products when compared to these gained after frying. 
Table 3. Total polyphenols content (TPC) $(n=2)$ and antioxidant capacity (Trolox equivalent antioxidant capacity (TEAC) ABTS and FRAP (Ferric Reducing Antioxidant Potential)) ( $n=4)$ of fried and microwaved snacks made from Jerusalem artichoke flour with addition of fruit powders during storage for 5 weeks.

\begin{tabular}{|c|c|c|c|c|c|c|c|c|}
\hline \multirow[t]{2}{*}{$\begin{array}{c}\text { Storage } \\
\text { Time (Week) }\end{array}$} & \multicolumn{2}{|c|}{ Control } & \multicolumn{2}{|c|}{ Cranberry } & \multicolumn{2}{|c|}{ Chokeberry } & \multicolumn{2}{|c|}{ Blackcurrant } \\
\hline & F & $\mathbf{M}$ & F & $\mathbf{M}$ & F & $\mathbf{M}$ & F & $\mathbf{M}$ \\
\hline \multicolumn{9}{|l|}{ ТРC $[\mu \mathrm{g} / \mathrm{g}]$} \\
\hline 0 & $694.13 \pm 18.38^{a, * * * *}$ & $745.28 \pm 16.97^{b, * * *}$ & $948.34 \pm 49.86^{\mathrm{d}, * * * * *}$ & $1012.82 \pm 16.97^{e, * * * *}$ & $946.86 \pm 44.40^{\mathrm{d}, * * * * * *}$ & $1062.76 \pm 14.14^{\mathrm{e}, * * * * *}$ & $825.97 \pm 48.87 \mathrm{c}, * * * * *$ & $968.18 \pm 5.66^{\mathrm{d}, * * * *}$ \\
\hline 1 & $672.97 \pm 2.83^{\mathrm{a}, * * * *}$ & $726.82 \pm 8.49^{b, * * *}$ & $724.30 \pm 36.41^{b, * * * *}$ & $935.87 \pm 7.07 \mathrm{~d}, * * * *$ & $799.19 \pm 8.49^{c, * * * * *}$ & $952.85 \pm 2.83^{\mathrm{d}, * * * *}$ & $785.33 \pm 7.07^{c, * * * *}$ & $936.99 \pm 8.49^{d, * * * *}$ \\
\hline 2 & $624.13 \pm 33.94^{\mathrm{a}, * *}$ & $688.57 \pm 11.311^{a, * *}$ & $646.00 \pm 14.14^{c, * * * *}$ & $860.93 \pm 13.86^{c, * *}$ & $744.75 \pm 2.83^{\mathrm{d}, * * * *}$ & $901.35 \pm 1.41^{\mathrm{d}, * * *, * * * *}$ & $592.11 \pm 2.83^{b, * * * *}$ & $843.31 \pm 15.56^{b, * * *}$ \\
\hline 3 & $587.60 \pm 26.87^{\mathrm{a}, * * *}$ & $656.33 \pm 8.49^{a} a^{*}, * *$ & $578.92 \pm 11.32^{a^{a}, * * *}$ & $851.08 \pm 19.80^{\mathrm{d}, * *}$ & $684.75 \pm 5.66^{\mathrm{b}, * * *}$ & $871.29 \pm 15.56^{\mathrm{d}, * *}$ & $560.76 \pm 14.14^{\mathrm{a}, *}$ & $769.86 \pm 14.14^{c, * *}$ \\
\hline 4 & $524.24 \pm 33.94^{\mathrm{b}, *}$ & $625.64 \pm 14.14^{\mathrm{c}, *}$ & $553.87 \pm 4.24^{b, *}$ & $836.66 \pm 8.49^{\mathrm{e}, * *}$ & $639.23 \pm 28.28^{c, * *}$ & $847.37 \pm 9.90^{\mathrm{e}, * *}$ & $455.62 \pm 7.07^{\mathrm{a}, *}$ & $741.05 \pm 1.41^{\mathrm{d}, * *}$ \\
\hline 5 & $489.08 \pm 12.72^{a, *}$ & $585.83 \pm 7.07^{c, *}$ & $513.87 \pm 18.38^{b, *}$ & $737.70 \pm 9.91 \mathrm{e}^{\mathrm{e}, *}$ & $591.05 \pm 1.42^{c, *}$ & $784.35 \pm 5.66^{\mathrm{f}, *}$ & $440.43 \pm 28.28^{a, *}$ & $639.77 \pm 14.15^{\mathrm{d}, *}$ \\
\hline \multicolumn{9}{|l|}{$\begin{array}{c}\text { TEAC ABTS } \\
{[\mu \mathrm{mol} \text { Trolox } / \mathrm{g}]}\end{array}$} \\
\hline 0 & $10.34 \pm 0.08^{a, * * * * *}$ & $13.01 \pm 0.01^{\mathrm{d}^{, * * * * * *}}$ & $13.10 \pm 0.08^{c, * * * * * *}$ & $15.30 \pm 0.08^{\mathrm{d}, * * * *}$ & $10.62 \pm 0.51^{b, * * * * *}$ & $12.38 \pm 0.07^{c, * * * * *}$ & $13.22 \pm 0.02^{c}{ }^{* * * * * * *}$ & $18.84 \pm 0.03^{e, * * * * *}$ \\
\hline 1 & $9.85 \pm 0.06^{\mathrm{a}, * * * * *}$ & $11.78 \pm 0.06^{c, * * * * *}$ & $12.93 \pm 0.04^{\mathrm{d}, * * * * *}$ & $15.27 \pm 0.06^{e, * * * * *}$ & $10.28 \pm 0.07^{b, * * * *}$ & $12.43^{ \pm} 0.03^{\mathrm{d}, * * * *}$ & $11.51 \pm 0.29^{c, * * * * *}$ & $17.72 \pm 0.02^{\mathrm{f}, * * * * *}$ \\
\hline 2 & $9.14 \pm 0.61^{\mathrm{a}, * * *}$ & $11.32 \pm 0.26^{c, * * * *}$ & $12.88 \pm 0.07^{\mathrm{e}, * * * *}$ & $15.11 \pm 0.09^{\mathrm{f}, * * * *}$ & $9.76 \pm 0.02^{b, * * * *}$ & $12.38 \pm 0.06^{\mathrm{d}, * * * * *}$ & $11.27 \pm 0.06^{c, * * * *}$ & $17.09 \pm 0.07 \mathrm{~g}, * * * *$ \\
\hline 3 & $8.69 \pm 0.34^{a, * * *}$ & $10.96 \pm 0.05^{c, * * *}$ & $12.61 \pm 0.08^{\mathrm{e}, * * *}$ & $13.84 \pm 0.03 \mathrm{f}_{, * *}$ & $9.32 \pm 0.02^{b, * *}$ & $11.69 \pm 0.07^{\mathrm{d}, * * * *}$ & $10.89 \pm 0.08^{c, * * *}$ & $16.29 \pm 0.07 \mathrm{~g}, * *$ \\
\hline 4 & $8.78 \pm 0.06^{\mathrm{a}, * * *}$ & $10.67 \pm 0.10^{c, * *}$ & $12.21 \pm 0.08^{\mathrm{e}, * *}$ & $13.69 \pm 0.16^{\mathrm{f}, * *}$ & $9.13 \pm 0.02^{b, *}$ & $11.47 \pm 0.04 \mathrm{~d}, * * *$ & $10.34 \pm 0.03^{c, * *}$ & $16.15 \pm 0.12 \mathrm{~g}, * * *$ \\
\hline 5 & $7.90 \pm 0.08^{a, *}$ & $10.25 \pm 0.08^{\mathrm{d}, *}$ & $11.76 \pm 0.05^{\mathrm{f}, *}$ & $13.35 \pm 0.04^{g, *}$ & $8.98 \pm 0.07^{b, *}$ & $11.16 \pm 0.13^{\mathrm{e}, *}$ & $9.48 \pm 0.07^{c, *}$ & $15.64 \pm 0.16^{\mathrm{h}, *}$ \\
\hline \multirow{2}{*}{\multicolumn{9}{|c|}{$\begin{array}{c}\text { FRAP } \\
{[\text { umol Trolox/g] }}\end{array}$}} \\
\hline & & & & & & & & \\
\hline 0 & $26.65 \pm 0.82^{\mathrm{a}, * * * *}$ & $37.06 \pm 0.05^{\mathrm{d}, * * * * *}$ & $28.92 \pm 0.02^{c, * * * * *}$ & $40.54 \pm 0.03^{\mathrm{d}, * * * *}$ & $27.96 \pm 0.08^{b, * * * *}$ & $38.52 \pm 0.02 \mathrm{e}, * * *$ & $27.18 \pm 0.16^{\mathrm{c}, * *}$ & $39.54 \pm 0.05^{\mathrm{f}, * * * *}$ \\
\hline 1 & $25.30 \pm 0.88^{\mathrm{a}, * * * * * * * *}$ & $36.95 \pm 0.04^{c, * * * *}$ & $27.42 \pm 0.34^{b, * * * *}$ & $39.45 \pm 0.16^{\mathrm{e}, * * *}$ & $25.80 \pm 0.08^{a, * * *}$ & $38.41 \pm 0.82 \mathrm{~d}, * * * *$ & $27.07 \pm 0.06^{b, * * * * * * *}$ & $39.08 \pm 0.65^{e, * * *}$ \\
\hline 2 & $24.84 \pm 0.93 \mathrm{a}, * * * *$ & $36.05 \pm 0.04 \mathrm{~d}, * * *$ & $26.03 \pm 0.82^{b, * * *}$ & $39.01 \pm 0.01 \mathrm{~g}, * * *$ & $25.36 \pm 0.05^{a, * * *}$ & $37.96 \pm 0.07 \mathrm{e}, * *$ & $26.92 \pm 0.82^{c, * *}$ & $38.38 \pm 0.09 \mathrm{f}, * *$ \\
\hline 3 & $24.02 \pm 0.02^{\mathrm{a}, * *}$ & $35.03 \pm 0.23 \mathrm{~d}_{, * *}$ & $25.92 \pm 0.02^{b, * *}$ & $38.85 \pm 0.06^{\mathrm{f}, * *}$ & $24.42 \pm 0.16^{\mathrm{a}, * *}$ & $37.68 \pm 0.09 \mathrm{e}, * *$ & $26.61 \pm 0.82^{c, * *}$ & $38.21 \pm 0.011_{, * *}$ \\
\hline 4 & $23.53 \pm 0.74^{\mathrm{a}, *}$ & $34.63 \pm 0.80^{c, *}$ & $25.53 \pm 0.24^{b, *}$ & $37.32 \pm 0.25^{\mathrm{d}, *}$ & $23.72 \pm 0.08^{a, *}$ & $37.40 \pm 0.06^{\mathrm{d}, * * *}$ & $25.63 \pm 0.16^{b, *}$ & $38.07 \pm 0.08^{\mathrm{e}, * *}$ \\
\hline 5 & $23.51 \pm 0.82^{a, *}$ & $34.61 \pm 0.01^{c, *}$ & $25.24 \pm 0.67^{b, *}$ & $37.26 \pm 0.21^{\mathrm{e}, *}$ & $23.68 \pm 0.08^{a, *}$ & $36.60 \pm 0.24 \mathrm{~d}^{\mathrm{d} *}$ & $25.44 \pm 0.36^{\mathrm{b}, *}$ & $37.42 \pm 0.02 \mathrm{e}^{\mathrm{e}, *}$ \\
\hline
\end{tabular}

F-fried, M-microwaved; values are means \pm standard deviation; different letters $(\mathrm{a}, \mathrm{b}, \mathrm{c}, \mathrm{d}, \mathrm{e}, \mathrm{f}, \mathrm{g})$ in rows and asterisk $(*, * * * * * * * * * * * * * *)$ in columns represent significant differences $(p \leq 0.05)$. 


\subsubsection{Antioxidant Capacity}

In the case of the control samples, similarly to total polyphenolic compounds content, the antioxidant capacity measured by the ability of extracts to scavenge the ABTS radical cations and to reduce the ferric ions was stronger for snacks gained after usage of microwaves (approximately $20.5 \%$ and $28 \%$, respectively) when compared to frying (Table 3 ).

Addition of fruit juice powders into the pellets has no influence on the antioxidant capacity values examined by TEAC ABTS assay when chokeberry powder was applied, whereas addition of blackcurrant and cranberry powders increased the TEAC ABTS values of, respectively, approximately $31 \%$ and $22 \%$ for microwaved samples, and $22 \%$ and $15 \%$ for snacks gained after frying. Camire et al. [35] showed that ability of extruded corn breakfast cereals to scavenge of ABTS radical cations decrease when the concord grape, cranberry, blueberry and red raspberry powders were applied for their preparation. Interestingly, the method of snacks production (microwaving/frying) had significant influence when the blackcurrant juice powder was added that caused a difference between TEAC ABTS values of almost 30\% between microwaved and fried samples. Addition of cranberry and chokeberry powders resulted, on average, in $14.3 \%$ differences, that pointed out a stronger influence of frying on blackcurrant powder's bioactive components. As the dominant group of polyphenols in this powder was anthocyanins, it can be concluded that frying caused stronger degradation of these constituents than application of microwaves at $700 \mathrm{~W}$. Storage resulted in a linear decrease in TEAC ABTS values up to 5 weeks, regardless of the type of powder added. The strongest degradation of compounds able to scavenge ABTS radicals was noted in case of snacks gained after frying with $3 \%$ addition of blackcurrant juice powders (almost 30\%) in comparison to non-stored products. On the other hand, in snacks gained after usage of microwaves, the decrease in TEAC ABTS values during 5-week storage was at the level of $17 \%$ which showed that frying had a stronger impact on the thermolability of bioactives in blackcurrant powder. In the case of the control samples, the decrease in antioxidant capacity values was approximately $22 \%$, regardless of the method used for snack preparation.

The addition of cranberry and chokeberry powders caused higher retention of compounds able to scavenge ABTS radical cations, thus products gained with application of these powders seems to be more stable during storage in terms of the degradation of bioactive constituents.

In the case of antioxidant capacity measured by the ability of extracts to reduce the ferric ions, the addition of chokeberry, blackcurrant and cranberry powders resulted in increase in FRAP values, respectively, of $3.8 \%, 6.3 \%$ and $8.6 \%$. Similarly to TEAC ABTS, the strongest impact was noted when the blackcurrant and cranberry powders were applied. Usage of microwaves at $700 \mathrm{~W}$ and frying also had a significant impact on the FRAP values of products obtained when the juice powders were added. The application of microwaves resulted in almost $29 \%$ higher values of FRAP when compared to samples gained after frying. This suggest a stronger degradation of compounds able to reduce the ferric ions during application of this type of pellets processing into snacks. A 5-weeks storage led to a slight linear decrease in the FRAP values for all analysed snacks. In products gained after application of microwaves the decrease was only, on average, $6.1 \%$ whereas for products gained after frying was $11.5 \%$ for snacks with fruit powders. The degradation of native components present in control samples (no fruit powders addition) during storage was at the same level (microwaves: down to 6.6\%; frying down to $12.7 \%$ ) which mean that native compounds present in pellets had a significant contribution to FRAP values during storage.

It was indicated that antioxidant capacity measured by two different in vitro assays was correlated with the content of total polyphenolic compounds ( $r=0.58$ for TEAC ABTS and $r=0.61$ for FRAP). A strong negative correlation was observed between browning index and TEAC ABTS $(r=-0.60)$ as well as FRAP $(r=-0.89)$. This indicated that antioxidant capacity of snacks during preparation and storage was depended more on native bioactive compounds than that formed during processing. 


\subsubsection{Oxidative and Hydrolytic Changes}

The fat absorbed by pellets during frying affects the ultimate properties of the finished products. Qualitative parameters of the oil contained in snacks depend mainly on the content of products of fat oxidation and hydrolysis. The oxidative changes of fat contained in fried snacks were monitored by determining the peroxide value, while hydrolytic — by acid value (Figure 2).

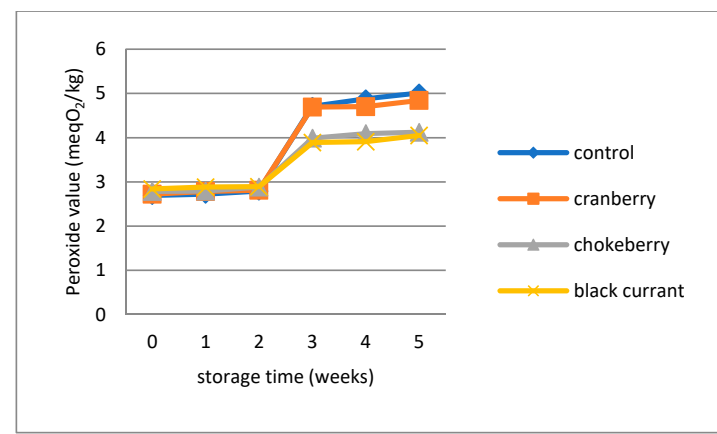

(a)

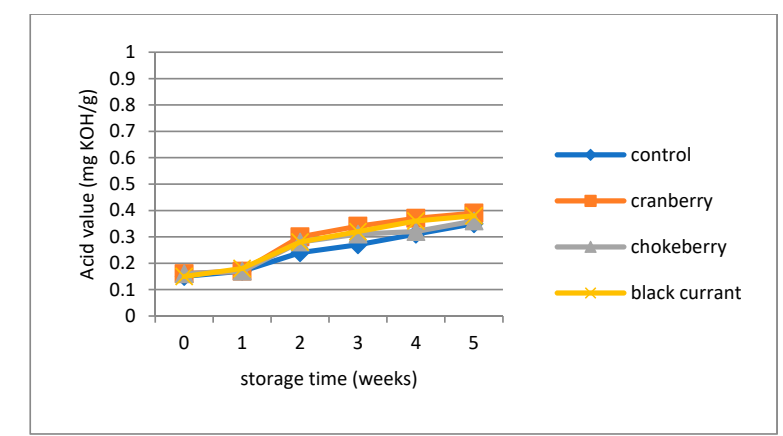

(b)

Figure 2. Changes of (a) peroxide value and (b) acid value of the fat extracted from the fried snacks during storage for 5 weeks.

Addition of fruit powders as a source of natural antioxidant compounds had a beneficial effect on inhibition of oxidative changes in fat contained in snacks however the extend of oxidative changes depended on type of fruit powder. The strongest effect was observed in samples with addition chokeberry and blackcurrant powders. Over the storage period, the peroxide value of fat of these snacks was $20-25 \%$ lower compared to the control sample. On the other hand, non-significant changes were observed between snacks with cranberry powder addition and control sample. It can be explained by different composition of powders and highest inhibition effect of polyphenolic compounds present in, both, chokeberry and blackcurrant powders. Such observations are in accordance with the others when different additives being a source of polyphenolic compounds or other antioxidants were added to snacks or potato chips $[7,28]$. Nemś at al. [24] showed that addition of dried potatoes of red or blue fleshed into pellets recipe increased storage stability of fried snacks by decreasing ratio of oxidative changes. Hydrolytic changes are more connected with water content in products. As moisture content increased with storage time the acid value increased in all analyzed samples however the rate of hydrolytic transformation was so low that the level of $2.00 \mathrm{mg} \mathrm{KOH} / \mathrm{g}$ suggested in the standard was not exceeded in any of the analyzed products [23].

\subsection{Sensory Analysis}

Newly designed products should have appropriate sensory features. The appearance, colour, taste, aroma and texture are particularly important quality distinguishing features in relation to snacks. These attributes depend on the additives added to the recipe as well as on technological parameters such as the expansion method. Fried snacks, independently on recipe, were higher evaluated as fresh products. Taste and smell as well as texture of snacks were more accepted when frying in oil was chosen as expansion method in comparison with microwaving. Visual aspects (Figure 1) also influenced the acceptability of ready to eat snacks. Fried snacks exhibited more delicate texture and were more wavy, while microwaved were more expanded with characteristic puffing effects. There were also observed differences in colour-especially influenced by the kind of fruit powder added. The most intensive colour had snacks with chokeberry powder addition and it was highly rated. However, during storage, because of oil deterioration mostly connected with oxidative changes, fried snacks lost their acceptability much faster than snacks with low fat content. Such phenomenon was observed according to control snacks as well as with cranberry powder addition, where peroxide value increased with a higher ratio (Figure 3 ) than in snacks with the addition of chokeberry and black currant powders. 
It can be explained by the different polyphenol composition of fruit powders and better antioxidant efficiency of compounds in chokeberry and black currant.
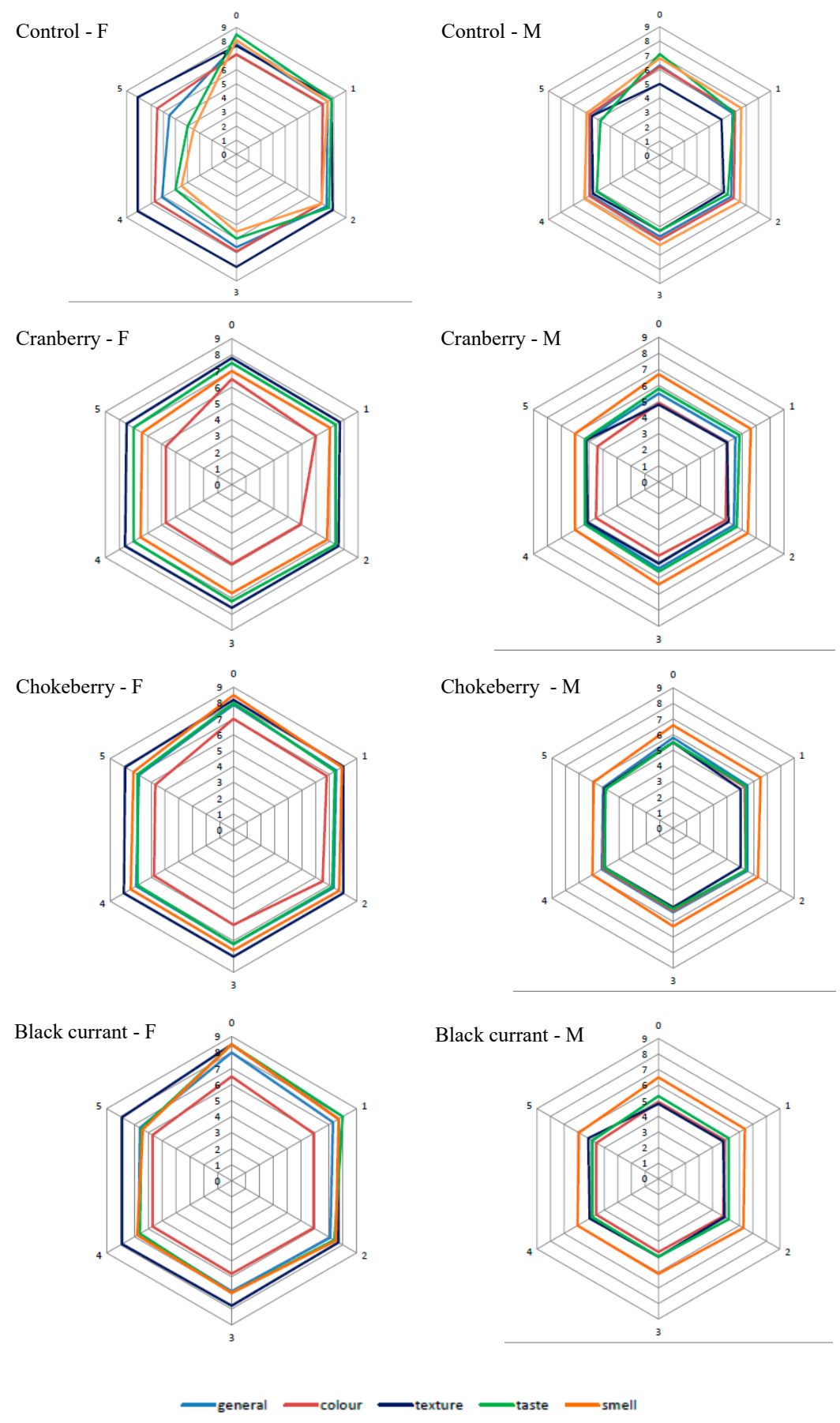

Figure 3. Sensory properties of fried (F) and microwaved (M) snacks made from Jerusalem artichoke flour with addition of fruit powders during storage for 5 weeks.

The most significant changes during storage were observed in decreasing of acceptability of smell and flavour (decrease up to 50\% in control sample and $10-20 \%$ in samples with fruit powders addition). On the other hand, microwaved snacks exhibited much more stable sensory quality during the 5-week period of storage. Within the storage time, the snacks texture deteriorated. The snacks lost their crunchiness and became harder. Kita [36] also noted that during the 5-month storage of wheat snacks, their sensory properties gradually deteriorated. Similar changes were noticed in snacks with 
various addition of cereal bran. Snacks with triticale bran were rated best, and rye bran the worst. Storage affected the deterioration of the consistency and smell, which was associated with increasing the moisture content of snacks [37].

\section{Conclusions}

The addition of fruit powders into snack compositions caused an increase in the content of total polyphenolic compounds and improved the antioxidant capacity of the products obtained. The addition of fruit powders influenced the colour as well as the texture of snacks. Products with fruit powders exhibited a more attractive colour, while their texture was harder in comparison to the control sample. A stronger effect was observed in snacks obtained by microwaving. Snacks with fruit powders addition exhibited better storage stability, what was especially important in the case of fried snacks where oxidative deterioration is most significant. The best effect was observed when chokeberry or blackcurrant powders were used as additives. Replacing frying by microwaving as the expansion method allowed one to obtain snacks with acceptable sensory attributes and stable quality during storage.

The addition of fruit juice powders into formulation used for snacks preparation is a promising approach towards the improvement of the functionally of ready-to-eat food products. Results obtained provide a clue for the design of the novel products, however, further research connected with the thorough examination of the alterations in single polyphenolic compounds are required.

Author Contributions: Conceptualization, A.K.; Investigation J.N. and A.M.-C.; writing—original draft preparation A.M.-C. and A.K. All authors have read and agreed to the published version of the manuscript.

Funding: This research received no external funding.

Acknowledgments: This research was supported by Food\&Health and Plant4Foods leading research groups.

Conflicts of Interest: The authors declare no conflict of interest.

\section{References}

1. Tumuluru, J.S. Snack foods: Role in diet. In Encyclopedia of Food and Health; Caballero, B., Finglas, P.M., Toldrá, F., Eds.; Academic Press: Oxford, UK, 2016; pp. 6-12. ISBN 978-0-12-384953-3.

2. Espinoza-Moreno, R.J.; Reyes-Moreno, C.; Milán-Carrillo, J.; López-Valenzuela, J.A.; Paredes-López, O.; Gutiérrez-Dorado, R. Healthy ready-to-eat expanded snack with high nutritional and antioxidant value produced from whole amarantin transgenic maize and black common bean. Plant Foods Hum. Nutr. 2016, 71, 218-224. [CrossRef] [PubMed]

3. Lachman, J.; Kays, S.J.; Nottingham, S.F. Biology and chemistry of Jerusalem Artichoke Helianthus tuberosus L. Biol. Plant 2008, 52, 492. [CrossRef]

4. Petkova, N.; Ivanov, I.; Denev, P.; Pavlov, A. Bioactive Substance and free radical scavenging activities of flour from Jerusalem Artichoke (Helianthus tuberosus L.) tubers-A comparative study. Turk. J. Agric. Nat. Sci. 2014, 1, 1773-1778.

5. Tchoné, M.; Bärwald, G.; Annemüller, G.; Fleischer, L. Separation and identification of phenolic compounds in Jerusalem Artichoke (Helianthus tuberosus L.). Sci. Aliment. 2006, 26, 394-408. [CrossRef]

6. Radovanovic, A.; Stojceska, V.; Plunkett, A.; Jankovic, S.; Milovanovic, D.; Cupara, S. The use of dry Jerusalem artichoke as a functional nutrient in developing extruded food with low glycaemic index. Food Chem. 2015, 177, 81-88. [CrossRef]

7. Pẹksa, A.; Kita, A.; Carbonell-Barrachina, A.A.; Miedzianka, J.; Kolniak-Ostek, J.; Tajner-Czopek, A.; Rytel, E.; Siwek, A.; Miarka, D.; Drożdż, W. Sensory attributes and physicochemical features of corn snacks as affected by different flour types and extrusion conditions. LWT Food Sci. Technol. 2016, 72, 26-36. [CrossRef]

8. Ruiz-Armenta, X.A.; Zazueta-Morales, J.D.J.; Delgado-Nieblas, C.I.; Carrillo-López, A.; Aguilar-Palazuelos, E.; Camacho-Hernández, I.L. Effect of the extrusion process and expansion by microwave heating on physicochemical, phytochemical, and antioxidant properties during the production of indirectly expanded snack foods. J. Food Process. Preserv. 2019, 43, e14261. [CrossRef] 
9. Potter, R.; Stojceska, V.; Plunkett, A. The use of fruit powders in extruded snacks suitable for Children's diets. LWT Food Sci. Technol. 2013, 51, 537-544. [CrossRef]

10. AOAC. Official Method 930.04_Loss on Drying (Moisture) in Plants. Available online: http://www. aoacofficialmethod.org/index.php?main_page=product_info\&cPath=1\&products_id=1228 (accessed on 13 July 2020).

11. PN-ISO 3960:2017 Animal and Vegetable Fats and Oils-Determination of Peroxide Value-Iodometric (Visual) Endpoint Determination. Available online: https://www.iso.org/standard/71268.html (accessed on 13 July 2020).

12. PN-EN ISO 660:2020 Animal and Vegetable Fats and Oils-Determination of Acid Value and Acidity. Available online: https://www.iso.org/standard/75594.html (accessed on 13 July 2020).

13. Kita, A.; Lisińska, G.; Gołubowska, G. The effects of oils and frying temperatures on the texture and fat content of potato crisps. Food Chem. 2007, 102, 1-5. [CrossRef]

14. Wrolstad, R.E.; Durst, R.W.; Lee, J. Tracking color and pigment changes in anthocyanin products. Trends Food Sci. Technol. 2005, 16, 423-428. [CrossRef]

15. Gao, X.; Björk, L.; Trajkovski, V.; Uggla, M. Evaluation of antioxidant activities of rosehip ethanol extracts in different test systems. J. Sci. Food Agric. 2000, 80, 2021-2027. [CrossRef]

16. Re, R.; Pellegrini, N.; Proteggente, A.; Pannala, A.; Yang, M.; Rice-Evans, C. Antioxidant activity applying an improved ABTS radical cation decolorization assay. Free Radic. Biol. Med. 1999, 26, 1231-1237. [CrossRef]

17. Benzie, I.F.F.; Strain, J.J. The ferric reducing ability of plasma (FRAP) as a measure of "antioxidant power": The FRAP assay. Anal. Biochem. 1996, 239, 70-76. [CrossRef] [PubMed]

18. Sánchez-Rodríguez, L.; Cano-Lamadrid, M.; Carbonell-Barrachina, Á.A.; Sendra, E.; Hernández, F. Volatile composition, sensory profile and consumer acceptability of hydroSOStainable table olives. Foods 2019, 8, 470. [CrossRef] [PubMed]

19. García-Segovia, P.; Urbano-Ramos, A.M.; Fiszman, S.; Martínez-Monzó, J. Effects of processing conditions on the quality of vacuum fried cassava chips (Manihot esculenta Crantz). LWT Food Sci. Technol. 2016, 69, 515-521. [CrossRef]

20. Kawas, M.L.; Moreira, R.G. Effect of degree of starch gelatinization on quality attributes of fried tortilla chips. J. Food Sci. 2001, 66, 300-306. [CrossRef]

21. Figiel, A.; Michalska, A. Overall quality of fruits and vegetables products affected by the drying processes with the assistance of vacuum-microwaves. Int. J. Mol. Sci. 2017, 18, 71. [CrossRef]

22. Dueik, V.; Robert, P.; Bouchon, P. Vacuum frying reduces oil uptake and improves the quality parameters of carrot crisps. Food Chem. 2010, 119, 1143-1149. [CrossRef]

23. Polish Standard PN-A-74780:1996 Potato Products. Fried Potato Snacks. Available online: https://sklep.pkn. pl/pn-a-74780-1996p.html (accessed on 1 July 2020).

24. Nemś, A.; Pęksa, A. Polyphenols of coloured-flesh potatoes as native antioxidants in stored fried snacks. LWT 2018, 97, 597-602. [CrossRef]

25. Pęksa, A.; Miedzianka, J.; Kita, A.; Czopek, A.T.; Rytel, E. The quality of fried snacks fortified with fiber and protein supplements. Potravin. Slovak J. Food Sci. 2010, 4, 59-64. [CrossRef]

26. Bhat, N.A.; Wani, I.A.; Hamdani, A.M.; Gani, A. Effect of extrusion on the physicochemical and antioxidant properties of value added snacks from whole wheat (Triticum aestivum L.) flour. Food Chem. 2019, 276, $22-32$. [CrossRef] [PubMed]

27. Peressini, D.; Foschia, M.; Tubaro, F.; Sensidoni, A. Impact of soluble dietary fibre on the characteristics of extruded snacks. Food Hydrocoll. 2015, 43, 73-81. [CrossRef]

28. Raleng, A.; Singh, A.; Chavan, P.; Attkan, A.; Singh, B. Standardization of deep-frying process and their effects on storage stability of pineapple pomace powder-incorporated rice-based extruded product. J. Food Process. Preserv. 2019, 43, e13950. [CrossRef]

29. Ngadi, M.; Li, Y.; Oluka, S. Quality changes in chicken nuggets fried in oils with different degrees of hydrogenatation. LWT Food Sci. Technol. 2007, 40, 1784-1791. [CrossRef]

30. Kerr, W. Chapter 9-Implications of non-equilibrium states and glass transitions in fried foods. In Non-Equilibrium States and Glass Transitions in Foods; Bhandari, B., Roos, Y.H., Eds.; Woodhead Publishing Series in Food Science, Technology and Nutrition; Woodhead Publishing: Cambridge, UK, 2017; pp. $209-239$. ISBN 978-0-08-100309-1. 
31. Cem, B.; Ali, E. Chips production from Jerusalem Artichoke (Helianthus tuberosus L.). Food Nutr. Sci. 2012, 3, 1321-1328. [CrossRef]

32. Pathare, P.B.; Opara, U.L.; Al-Said, F.A.-J. Colour measurement and analysis in fresh and processed foods: A review. Food Bioprocess. Technol. 2013, 6, 36-60. [CrossRef]

33. Michalska, A.; Wojdyło, A.; Majerska, J.; Lech, K.; Brzezowska, J. Qualitative and quantitative evaluation of heat-induced changes in polyphenols and antioxidant capacity in Prunus domestica L. by-products. Molecules 2019, 24, 3008. [CrossRef]

34. Michalska, A.; Wojdyło, A.; Lech, K.; Łysiak, G.P.; Figiel, A. Physicochemical properties of whole fruit plum powders obtained using different drying technologies. Food Chem. 2016, 207, 223-232. [CrossRef]

35. Camire, M.E.; Dougherty, M.P.; Briggs, J.L. Functionality of fruit powders in extruded corn breakfast cereals. Food Chem. 2007, 101, 765-770. [CrossRef]

36. Kita, A. Wpływ wybranych parametrów technologicznych na jakość smażonych produktów przekąskowych. Zesz. Nauk. Akad. Rol. We Wroctawiu 2006, 240, 1-65.

37. Gambuś, H.; Golachowski, A.; Bala-Piasek, A.; Nowotna, A.; Surówka, K.; Mikulec, A.; Bania, M. Ocena jakości ekstrudowanych chrupek z otrąb zbożowych. Żywność. Nauka. Technol. Jakość 2000, 4, 54-63.

(C) 2020 by the authors. Licensee MDPI, Basel, Switzerland. This article is an open access article distributed under the terms and conditions of the Creative Commons Attribution (CC BY) license (http://creativecommons.org/licenses/by/4.0/). 